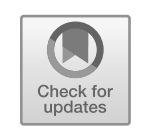

\title{
4 Elemente struktureller Studierbarkeit
}

In diesem Kapitel stehen die Elemente struktureller Studierbarkeit im Mittelpunkt, welche die Studierbarkeit für die in Kapitel 3 genannten Studierendengruppen beeinflussen können. Durch die Elemente innerhalb eines Studienganges können Hochschulen steuern, wann und wo Lehrveranstaltungen stattfinden und wie die Studierenden ihr Selbststudium gestalten. Die Elemente werden in verschiedenen Studienformaten (z. B. Teilzeitstudium, Fernstudium) unterschiedlich eingesetzt. Die Studienformate steuern dabei, wie viel Zeit Studierende für das Absolvieren des Studiums zur Verfügung haben und an welchem Lernort sie primär studieren.

Das Kapitel beginnt mit Strukturelementen, die die zeitliche Gestaltung des Studiums beeinflussen, und fährt mit dem Thema Blended-Learning fort, welches neben der zeitlichen Komponente auch den Lernort einbezieht. Im Anschluss werden vier Studienformate mit unterschiedlichen Studiengeschwindigkeiten und Lernorten vorgestellt.

Neben den hier vorgestellten Faktoren existieren weitere strukturelle Elemente, die das Studierverhalten beeinflussen, allerdings weniger strukturell und systematisch verankert (z. B. Beratung durch Lehrende) oder auf Hochschulebene festgelegt sind (z. B. Beurlaubungsregelungen, Fristen). Diese Elemente gehen nicht in die quantitative Analyse struktureller Studierbarkeit ein, da sie wegen der Festlegung auf Hochschulebene in den beiden untersuchten Hochschulen kaum variieren. Zur Erfassung der strukturellen Studierbarkeit sind sie dennoch interessant und können in einer Dokumentenanalyse erfasst werden. Ein Analyseschema für eine solche Dokumentenanalyse struktureller Studierbarkeit ist bei Buß et al. (2015) nachzulesen.

\subsection{Zeitliche Lage der Lehrveranstaltungen}

Die zeitliche Lage der Lehrveranstaltungen beeinflusst das Studierverhalten der Studierenden und die Studierbarkeit von Studiengängen stark. So wirken sich Überschneidungen zwischen Lehrveranstaltungen oder Veranstaltungen zu „unpassenden“ Zeiten negativ auf die Studierbarkeit aus (Kuhlee et al. 2009, S. 23; Schlögl und Neubauer 2006, S. 8).

Die zeitliche Lage der Lehrveranstaltungen kann von Hochschulen durch mindestens drei Strukturelemente gestaltet werden. Erstens können Hochschulen bei Zeiträumen von Lehrveranstaltungen auf die zeitlichen Bedarfe der Studierenden Rücksicht nehmen. Dies können beispielsweise freie Tage für Erwerbstätigkeit oder Lehrveranstaltungszeiten während der Betreuungszeiten für 
Kinder sein. Der Zeitpunkt einer Lehrveranstaltung beeinflusst das Studierverhalten. So zeigen Arulampalam et al. (2012, S. 369), dass zu unpassenden Zeiten am Vormittag und an bestimmten Wochentagen Studierende häufiger abwesend sind. Da Studierende unterschiedliche Bedarfe haben, ist die Definition von passenden Zeiträumen jedoch eine große Herausforderung (siehe dazu auch Kapitel 1 und Kapitel 8.1). Zweitens können Hochschulen die Intervalle der Lehrveranstaltungen festlegen. Das weit verbreitete Intervall ist der wöchentliche Turnus während der Vorlesungszeit. Eine weitere Möglichkeit ist die Blockung von Lehrveranstaltungen, wodurch die Studierenden seltener und dafür für längere Abschnitte an die Hochschule kommen. Mehrere Hochschulen haben in diesem Sinne Blockwochen oder Blocktage eingeführt (z. B. Hochschule Stralsund 2017). Der Vorteil von Blockveranstaltungen ist die Verdichtung der Veranstaltungszeit an bestimmten Tagen und die Reduktion der wöchentlich stattfindenden Veranstaltungen. Dies kann zu einer höheren zeitlichen Flexibilität führen, wenn die Blocktage oder Blockwochen frühzeitig bekannt gegeben werden (Buß et al. 2017b, S. 3). Blockveranstaltungen können jedoch im Vergleich zu wöchentlichen Veranstaltungen auch nachteilig sein, wenn Studierende aufgrund von Krankheit oder anderen Verpflichtungen große Teile eines Moduls verpassen. Ein drittes Element ist die Verlagerung von festgelegten Veranstaltungszeiten in das virtuelle Lernen (siehe Kapitel 4.3).

Studiengänge haben die Möglichkeit, inhaltlich gleiche Veranstaltungen zu unterschiedlichen Zeiten und mit unterschiedlichen Intervallen anzubieten und damit zeitliche Wahlmöglichkeiten zu schaffen. Dies wird von berufstätigen Studierenden und Studierenden mit Kind als positiv für die Vereinbarkeit wahrgenommen (Husemann und Müller 2018, S. 264 f.). Es setzt allerdings große Studiengänge oder Studiengänge mit kleinen Gruppengrößen voraus, bei denen Veranstaltungen aufgrund von großen Kohorten geteilt werden.

\subsection{Pflicht- und Wahlmodule}

Studierende verfügen üblicherweise über gewisse Freiheiten in der inhaltlichen Gestaltung des Studiums. Diese Gestaltungsmöglichkeit können Studierende durch die Auswahl von Wahlmodulen nutzen. Studien zu nicht-traditionellen Studierendengruppen nennen Wahlangebote als wichtiges Steuerungselement, wenn es darum geht, den eigenen Interessen nachgehen und zeitlich $\mathrm{zu}$ den anderen Verpflichtungen passende Module wählen zu können (Buß, Erbsland et al. 2018, S. 125; Schwikal et al. 2018, S. 308). Eine Übersicht über die Bedarfe an Wahlmöglichkeiten von unterschiedlichen Studierendengruppen ist in Kapitel 1 zu finden. 
Wahlmöglichkeiten eröffnen zeitliche und inhaltliche Flexibilität. Aus wie vielen Modulen Studierende wählen können und wie breit das inhaltliche Angebot damit ist, differiert (Kerres und Schmidt 2011, S. 177 ff.). Dabei spielt auch eine Rolle, ob die Wahlveranstaltungen nur für einen Studiengang angeboten werden und damit durch das Lehrdeputat des Studiengangs komplett abgedeckt werden müssen. Dies kann bei Studiengängen mit kleinen Kohorten zu einem begrenzten Wahlangebot führen (Röbken 2012, S. 242). Alternativ öffnen Hochschulen Lehrveranstaltungen anderer Fakultäten für alle Studierenden, wodurch den Studierenden eine inhaltlich breitere Auswahl an Veranstaltungen zur Verfügung steht. Wahlmöglichkeiten können in unterschiedlichen inhaltlichen Bereichen geschaffen werden. Sie können innerhalb des Studienfaches liegen, fachfremde Inhalte (Röbken 2012, S. 243) vermitteln oder im Bereich der Schlüsselkompetenzen angesiedelt sein. ${ }^{6}$

\title{
4.3 Blended-Learning und Lernorte
}

Virtuelle Lehre ist eine häufig genanntes Strukturelement, mit dem die Lernorte und Lernzeiten von Studierenden flexibler gestaltet werden sollen (Kerres 2012, S. 45; Koper 2015, S. 312; Kreidl 2011, S. 15; Zumbach und Astleitner 2016, S. 149). Dabei findet E-Learning komplett (online) am Computer oder anderen Endgeräten statt, wohingegen Blended-Learning Lehrveranstaltungen und ELearning an der Hochschule miteinander kombiniert (Thorne 2003, S. 16; Zumbach und Astleitner 2016, S. 146 f.). Im Blended-Learning werden also einige der Präsenzveranstaltungen durch virtuelle Lehre ersetzt (Schulmeister 2006, S. 192). Sauter et al. (2003, S. 68) definieren Blended-Learning wie folgt:

\begin{abstract}
„Blended Learning ist ein integriertes Lernkonzept, das die heute verfügbaren klassischen Lehrmethoden und -medien in einem sinnvollen Lernarrangement optimal nutzt. Es ermöglicht Lernen, Kommunizieren, Informieren und Wissensmanagement, losgelöst von Ort und Zeit in Kombination mit Erfahrungsaustausch, Rollenspiel und persönlichen Begegnungen im klassischen Präsenztraining.“
\end{abstract}

Die virtuelle Lehre kann für alle Teilnehmenden zeitlich parallel und damit synchron durchgeführt werden, oder aber sie ist asynchron organisiert und lässt unterschiedliche Bearbeitungszeitpunkte zu. Die zeitliche Flexibilisierung ist dabei am höchsten, wenn der virtuelle Anteil asynchron stattfindet. Synchrone Anteile werden beispielsweise durch Chats oder die Nutzung virtueller

\footnotetext{
${ }^{6}$ Beispielsweise sind diese drei Optionen im Bachelor-Studiengang BWL der Universität Göttingen möglich: https://www.uni-goettingen.de/de/23191.html.
} 
Klassenzimmer mit Präsentationen und Diskussionen forciert, da in diesem Fall alle Beteiligten zeitgleich online sein müssen.

Mehrere Studien zeigen eine hohe Zufriedenheit von Studierenden und Lehrenden mit Blended-Learning, wenn hierdurch die Vorteile von virtueller Lehre und Präsenzlehre verbunden werden können (Übersicht siehe Garrison und Vaughan 2008, 4 f.). In dieser Arbeit steht das Blended-Learning im Mittelpunkt, da die komplette Verlagerung der Lehre in den virtuellen Raum auf große Widerstände trifft und zahlreiche Nachteile mit sich bringt. Nachfolgend seien zunächst einige der Vorteile virtueller Lehre genannt. So können Studierende ihrem eigenen Lerntempo folgen; Lehrinhalte und Lernmaterialien sowie schriftliche Diskussionen sind lange verfügbar und transparent (Zumbach und Astleitner 2016, S. 149 ff.). Außerdem können Lehrende auf eine umfassende Palette an Methoden zurückgreifen, die auch in Veranstaltungen mit einer großen Anzahl an Studierenden realisierbar sind. Vorteile von Präsenzlehre hingegen sind unter anderem der soziale Kontakt zu Lehrenden und Studierenden und die unmittelbare Kommunikation (Arnold et al. 2004, S. 19 ff.). Die Nachteile oder Beschränkungen von Blended-Learning analysiert unter anderem Uhl (2003, S. 49 ff.). Er zeigt anhand von E-Learning-Projekten an acht Hochschulen, dass die Studierenden die Onlineplattformen neben Büchern und Zeitschriften primär als weitere Quelle zur Vor- und Nachbereitung nutzen, obwohl sie deutlich mehr Möglichkeiten bietet. Die Studierenden fordern weiterhin Präsenzveranstaltungen, da ihnen die Kommunikation und der direkte soziale Kontakt mit Kommilitoninnen und Kommilitonen und Lehrenden wichtig erscheinen. Viele Studierende geben außerdem an, dass die selbstgesteuerte Gestaltung der Lernprozesse für sie eine Herausforderung darstellt (Concannon et al. 2005, S. 507).

Der Erfolg von Blended-Learning hängt trotz dieser generellen Vor- und Nachteile stark vom konkreten Design der virtuellen- und Präsenzlernphase ab. Beim Design des Blended-Learning (Ablauf, Aufteilung zwischen Präsenz- und Onlinephasen, Auswahl der Methodik und Tools) ist insbesondere zu beachten, dass es zu den Lernzielen der Lehrveranstaltung passt. Die didaktische Konzeption und das darin berücksichtigte positive Feedback durch Lehrende ist einer der wichtigsten Faktoren, mit denen die Akzeptanz durch die Studierenden gewährleistet wird (Concannon et al. 2005, S. 508 f.; Kreidl 2011, S. 142).

Wenn sich Lehrveranstaltungen aus didaktischer Sicht für Blended-Learning eignen, sind vor der Implementierung noch rechtliche Vorgaben und das Vorhandensein ausreichender Ressourcen zu prüfen. So erlauben es die Lehrverpflichtungsverordnungen der einzelnen Bundesländer nicht gleichermaßen, Semesterwochenstunden statt im Hörsaal in virtueller Form zu erbringen (Lungershausen et al. 2016, S. 102 ff.). Schließlich bedeutet die 
Entwicklung von virtueller Lehre einen nennenswerten Ressourcenaufwand finanzieller und personeller Art, der für einen nachhaltigen Erfolg langfristig gesichert sein sollte (Bremer et al. 2010, S. 8ff.).

Zusammenfassend kann man konstatieren, dass Blended-Learning zu einer hohen zeitlichen und örtlichen Flexibilisierung des Studiums führen kann. Um gleichzeitig eine hohe didaktische Qualität und gute Lernerfolge zu sichern, bedarf es der Medienkompetenzen von Studierenden und Lehrenden (Baacke 1996, S. 8 ff.; Horvath 2009, S. 2), einer guten didaktischen Konzeption des Angebots sowie technischer und personeller Ressourcen für die nachhaltige und reibungsarme Umsetzung.

\subsection{Studienformate}

Als Studienformate gelten die unterschiedlichen Typen von Studiengängen, die gegenüber dem Vollzeitstudium andere Studiengeschwindigkeiten vorsehen, andere Lernorte integrieren oder parallele Berufstätigkeit ermöglichen. Bargel und Bargel (2014, S. 27) nennen dabei fünf offizielle Formen: Teilzeitstudium, Fernstudium, duales Studium, berufsbegleitendes Studium und Weiterbildung. Die Abgrenzungen zwischen den Studienformaten werden je nach Autorin oder Autor unterschiedlich gezogen. Die meisten Definitionen sind sich jedoch dahingehend einig, dass Fern- und berufsbegleitende Studiengänge im Voll- und Teilzeitformat angeboten werden können, wohingegen duale Studiengänge in der Regel Vollzeitstudiengänge sind (Hochschulrektorenkonferenz 2016, S. 3; Statistische Ämter des Bundes und der Länder 2017, S. 71).

Die genannten Studienformate werden nachfolgend definiert und bezogen auf ihren Entwicklungsstand in Deutschland, ihre Nachfrage und gesetzliche Vorgaben analysiert. Dabei wird das berufsbegleitende Studium nicht gesondert dargestellt, sondern unter dem Teilzeitstudium subsumiert. Da der Fokus dieser Arbeit auf grundständigen und konsekutiven Studiengängen liegt, wird auf die Weiterbildung nicht näher eingegangen. Tabelle 11 bietet einen Überblick über die zahlenmäßige Aufteilung der Studierenden auf die unterschiedlichen Studienformate im Wintersemester 2016/2017 sowie die Anzahl an Studiengängen im November 2017 in Deutschland. 
Tabelle 11 Studierende nach Studienformaten im WiSe2016/17 und Studiengänge. Eigene Darstellung. Quellen: Statistisches Bundesamt (2017a), Statistisches Bundesamt (2017b), Hochschulkompass

\begin{tabular}{lrrrrr}
\hline & & \multicolumn{4}{c}{ davon... } \\
& Gesamt & Vollzeit & Teilzeit & Dual & Fernstudium* \\
\hline Studierende & 2.807 .010 & 2.513 .002 & 201.541 & 92.467 & 163.653 \\
\hline $\begin{array}{l}\text { Studiengänge } \\
(11 / 2017)\end{array}$ & 19.203 & 17.310 & 2.425 & 1.148 & 562 \\
\hline
\end{tabular}

* Fernstudierende können sowohl Vollzeit- als auch Teilzeit- und dual Studierende sein

\subsubsection{Vollzeitstudium}

Das Vollzeitstudium ist die häufigste Studienform; man geht dabei davon aus, dass die Studierenden zeitlich und örtlich ihren Hauptfokus auf das Studium an einem bestimmten Studienort legen. So präzisieren Klumpp und Rybnikova (2010, S. 25) das Studienformat als ,vollzeitiges Präsenzstudium“. Das Statistische Bundesamt fokussiert die „Konzeption der Bildungsprogramme für eine Voll- oder Teilzeitteilnahme" und beschreibt damit insbesondere die Gestaltung des Curriculums und der zeitlichen Anforderungen an ein Studium (Statistische Ämter des Bundes und der Länder 2017, S. 134). Auch die Anforderungen an Studiengänge durch die Akkreditierung und die darin enthaltenen Definitionen von Workload sowie fast alle gesetzlichen Vorgaben gehen von Vollzeitstudiengängen aus - es sei denn, sie formulieren explizit Ausnahmen (z. B. Akkreditierungsrat 2013). Nachfolgend liegt der Fokus auf dem zeitlichen Aspekt des Vollzeitstudiums und nicht auf dem Studienort des Präsenzstudiums, da viele Hochschulen durchaus Fernstudiengänge in Vollzeit anbieten.

Das Vollzeitstudium kann immer noch als Standard in der deutschen Hochschullandschaft eingestuft werden, da laut Hochschulkompass im November 2017 rund 90 Prozent aller Studiengänge im Vollzeitmodus angeboten wurden (17.310 Studiengänge). Dies bildet auch den Anteil der in diesen Studiengängen eingeschriebenen Studierenden ab, da 89,5\% der Studierenden im Wintersemester 2016/17 in Vollzeitstudiengängen eingeschrieben sind (Statistisches Bundesamt 2017a). Die 21. Sozialerhebung geht mit 97 \% sogar von höheren Zahlen aus (Middendorff et al. 2017, S. 15). Die nachfolgenden Beschreibungen der Studienformate sind immer in Abgrenzung zum Standard des Vollzeitstudiums zu sehen. 


\subsubsection{Teilzeitstudium}

Teilzeitstudium wurde schon in den 1980er Jahren entwickelt und in einem umfassenden Modellversuch im Land Baden-Württemberg 2002/2003 ausgebaut, findet aber erst im Zuge des Bologna-Prozesses und der Umstellung auf die Bachelor- und Masterstudiengänge größere politische Aufmerksamkeit (Bargel und Bargel 2014, S. 9 ff.; Hochschulrektorenkonferenz 2014, S. 2). 2016 forderte die Hochschulrektorenkonferenz die Hochschulen dazu auf, vermehrt das Teilzeitstudium zu ermöglichen und somit flexible Studienstrukturen zu schaffen (Hochschulrektorenkonferenz 2016, S. 5 ff.). Ziel dieser Bemühungen ist es, insbesondere im Bachelorbereich die Studiengänge für erwerbstätige Studierende oder Studierende mit Familienpflichten zu öffnen. Die HRK verweist daher auf die Verzahnung mit der Anrechnung außerhochschulischer Leistungen und Blended-Learning, um eine hohe Flexibilität zu schaffen. Ein Teilzeitstudium kann nach Bargel $(2013$, S. 7) in vier unterschiedliche Varianten eingeteilt werden:

1. Informelles Teilzeitstudium: Studierende sind in Vollzeitstudiengängen eingeschrieben und können das Studium eigenständig an ihre zeitlichen Bedürfnisse anpassen. Hierbei müssen sie teilweise Beeinträchtigungen durch Fristen o. Ä. in Kauf nehmen.

2. Entfristetes Teilzeitstudium: Studierende sind in Vollzeitstudiengängen eingeschrieben, können dieses Studium allerdings durch Fristverlängerungen offiziell strecken.

3. Individualisiertes Teilzeitstudium: die Anforderungen eines Vollzeitstudiums (ECTS, Workload) bestehen bleiben, aber der Studienverlauf kann in Absprache mit den an der Hochschule Zuständigen individuell gestaltet werden.

4. Formales Teilzeitstudium: angeboten wird ein gesonderter Studiengang oder eine Variante des Vollzeitstudiengangs mit eigenen Prüfungsmodalitäten. Dabei wird die Regelstudienzeit bis hin zur Verdoppelung gestreckt.

Im November 2017 wurden laut dem Hochschulkompass 2.425 Studiengänge in Deutschland als Teilzeitstudiengänge angeboten, wobei etwa 60 Prozent hiervon mit dem Master abschließen. Die Mehrzahl stellt eine Variante des parallel angebotenen Vollzeitstudiums dar. Dies ist möglich, da fast alle Landeshochschulgesetze ihren Hochschulen erlauben, die Studiengänge ebenfalls in Teilzeit anzubieten (z. B. Freie und Hansestadt Hamburg 2014, § 36; Land Baden-Württemberg 2014, § 30). Dabei unterscheiden sich die Bundesländer darin, ob sie, wie Baden-Württemberg, auch ein individuelles Teilzeitstudium 
ermöglichen oder, wie im Falle des Landes Hessen, ein formales Teilzeitstudium mit einem Umfang von 50 Prozent fordern (Land Hessen 2010, § 9).

Die fast flächendeckende Regelung des Teilzeitstudiums ${ }^{7}$ in den Hochschulgesetzen entspricht dem von den Studierenden angezeigten Bedarf an einem flexiblen Studium (Maschwitz und Brinkmann 2015, S. 58). So wenden 29 Prozent der Studierenden weniger als 25 Stunden in der Woche für ihr Studium auf und studieren damit de facto in Teilzeit (Middendorff et al. 2017, S. 58). Der 12. Studierendensurvey befragte Studierende danach, ob Hochschulen spezielle Studiengänge für Teilzeitstudierende entwickeln sollen. Dies bestätigten 21 Prozent der Universitäts- und 31 Prozent der Fachhochschulstudierenden. Betrachtet man die Fächergruppen, ist die Zustimmung in den Sozialwissenschaften (29 Prozent Universitäten, 38 Prozent FHs) besonders hoch, in den Naturwissenschaften (15 Prozent, Universität) und Ingenieurswissenschaften (18 Prozent, FH) besonders niedrig (Ramm et al. 2014, S. 450). Bargel und Bargel (2014, S. 220 ff.) gehen allerdings von einer gesicherten Mindestnachfrage von lediglich 6 Prozent aller Studierenden am formalen Teilzeitstudium aus, da viele Studierende trotz hoher Erwerbstätigkeit und weiterer Pflichten keine passenden, ausreichend flexiblen Angebote auffinden. Elf Prozent der im Studierendensurvey befragten Studierenden erwägen die Wahl eines formellen Teilzeitangebotes, wenn dieses bedarfsgerecht ausgelegt ist (Standardnachfrage). 16 Prozent möchten unter günstigen Rahmenbedingungen ein formelles Teilzeitangebot vielleicht nutzen, wenn es flexibel gestaltet ist (Maximalnachfrage).

Trotz der hohen Anzahl an angebotenen Teilzeitstudiengängen und der Prognosen studieren nur wenige Studierende offiziell in Teilzeit. Die Datenlage ist allerdings aufgrund der unterschiedlichen Erhebungsmethodiken und Definitionen des Teilzeitstudiums uneindeutig. Laut der 21. Sozialerhebung geben nur zwei Prozent der Studierenden an, in einem Teilzeitstudiengang eingeschrieben zu sein. An Fachhochschulen ist dieser Wert mit fünf Prozent etwas höher. Ein Prozent der Befragten sind in einem Vollzeitstudiengang mit Teilzeitoption eingeschrieben (Middendorff et al. 2017, S. 15). Bei der Interpretation der Daten ist $\mathrm{zu}$ berücksichtigen, dass Studierende von Fernhochschulen nicht an der Sozialerhebung teilnehmen, obwohl viele der zahlenmäßig besonders erfolgreichen Teilzeitstudiengänge an Fernhochschulen angeboten werden (Bargel und Bargel 2014, S. 56). Die H201-Daten des Statistischen Bundesamtes vom Wintersemester 2016/17 gehen von etwas höheren Einschreibezahlen aus (Statistisches Bundesamt 2017a). So studieren 7,1 Prozent aller eingeschriebenen Studierenden in Teilzeit; dies umfasst alle

\footnotetext{
${ }^{7}$ Alle Bundesländer außer Rheinland-Pfalz erwähnen im Jahr 2017 das Teilzeitstudium in ihren Hochschulgesetzen.
} 
Studiengänge „die nach Dauer und Unterrichtsbelastung eine studienbegleitende Berufstätigkeit zulassen“" (Statistische Ämter des Bundes und der Länder 2017, S. 134). Duale Studiengänge und strukturierte Promotionsprogramme sind nicht inbegriffen, Studierende von Weiterbildungs- sowie Fernstudiengängen und freie Promotionsstudierende zählen jedoch zu den Teilzeitstudierenden.

Insgesamt ist der Anteil an Teilzeitstudierenden an Fernhochschulen, im Masterbereich und in den Fächern der Wirtschaftswissenschaft besonders hoch (Bargel und Bargel 2014, 47 ff.). Somit bewegen sich die Einschreibungen in Teilzeitstudiengänge derzeit im Bereich der prognostizierten Mindestnachfrage. Die Prognosen weisen auf ein wichtiges Erfolgskriterium für Teilzeitstudiengänge hin: sie müssen die strukturellen Bedarfe der Studierenden tatsächlich erfüllen, da die Studierenden sonst auf Vollzeit- oder berufsbegleitende Studiengänge ausweichen und diese de facto in Teilzeit studieren (Maschwitz und Brinkmann 2015, $60 \mathrm{ff}$.).

Doch warum sind die formalen Teilzeitstudiengänge wenig attraktiv für Studierende mit zeitlichen Restriktionen? Zunächst haben Teilzeitstudierende kein Anrecht auf BAföG. Dies ist zwar für einen Anteil der Zielgruppe irrelevant, wenn die Studierenden einer (qualifizierten) Berufstätigkeit nachgehen. Verdienen die Studierenden in ihrer Erwerbstätigkeit allerdings nicht genügend oder sind Pflegeaufgaben, Beeinträchtigungen oder Engagement die Gründe für ihr Interesse an einem Teilzeitstudium, so ist das fehlende BAföG ein wichtiger Faktor bei der Studienentscheidung. Auch andere finanzielle Regelungen (Krankenversicherung, Steuerrecht, Rentenversicherung etc.) orientieren sich am Vollzeitstudierenden (Technische Universität Darmstadt 2017). Darüber hinaus beeinflussen besonders strukturelle Gründe die Attraktivität von Teilzeitstudiengängen. So ist die Beantragung oft recht aufwendig: Studierende müssen die Teilzeitoption in jedem Semester neu beantragen oder sich für einen längeren Zeitraum festlegen (Maschwitz und Brinkmann 2015, 65 f.; Steinhardt 2011a, 45 f.). Außerdem führt die Einschreibung in ein Teilzeitstudium oft nicht zu der erhofften Flexibilität - unter anderem deshalb, weil in vielen Bundesländern und Hochschulen nur Veranstaltungen im Umfang der Hälfte der üblichen ECTS (15 ECTS) belegt werden dürfen. Im Rahmen einer Institutionenstudie an der Universität Oldenburg und an der Hochschule Magdeburg-Stendal kristallisieren sich in diesem Kontext zwei Erfolgsfaktoren heraus. Erstens sollte die Anzahl der zu erwerbenden Kreditpunkte höher als 15 ECTS pro Semester liegen, da die meisten Studierenden ihre Studiendauer nicht verdoppeln möchten. Zweitens ist das Teilzeitstudium dann besonders interessant, wenn auch die Lehrveranstaltungszeiten flexibel sind, beispielsweise durch Blended-Learning oder durch mehrfach angebotene Veranstaltungen zu unterschiedlichen Zeiten (Buß et al. 2017a, S. 2 ff.). Letzteres ist jedoch nur in größeren Studiengängen 
möglich. Teilzeitstudiengänge dürfen in mehreren Bundesländern nur in zulassungsfreien Studiengängen angeboten werden (z. B. Land Hessen 2010). Dies schränkt die Fächerauswahl ein und führt bei einer Änderung der Zulassungsbeschränkung zu Unklarheiten. Schließlich ist das formale Teilzeitstudium unter den Studierenden oft wenig bekannt, sodass potenzielle Interessierte ihre Möglichkeiten nicht kennen (Steinhardt 2011a, S. 45). Bargel und Bargel (2014, S. 160) fassen eine Befragung unter den Hochschulen in BadenWürttemberg zu Erfolgsfaktoren für ein Teilzeitstudium zusammen und nennen über die schon genannten Punkte hinaus eine gute Betreuung und Unterstützung der Studierenden, den Einbezug der Berufswelt und eine hohe Lehrqualität.

Zusammenfassend lässt sich festhalten, dass ein flexibles formales oder individualisiertes Teilzeitstudium insbesondere berufstätigen Studierenden, aber auch Studierenden mit Sorgeaufgaben und Beeinträchtigungen Zugang zur Hochschule verschaffen und zu einer guten Vereinbarkeit beitragen kann. Dabei ist einschränkend zu beachten, dass beim formalen Teilzeitstudium kein BAföGAnspruch besteht.

\subsubsection{Fernstudium}

Beim Fernstudium handelt es sich um Studiengänge, in denen der überwiegende oder komplette Teil des Studiums zeit- und ortsunabhängig stattfindet. Die Studierenden lernen am Ort ihrer Wahl und kommen lediglich für kürzere Präsenzphasen oder Prüfungen an die (Fern-) Hochschulen oder ihre dezentral im Land verteilten Studienzentren. Das Fernstudium existiert schon seit mehr als 140 Jahren; 1875 wurde in Pretoria (Südafrika) die erste Fernuniversität gegründet (Zawacki-Richter 2013, 3 f.). Nach Garrison (1985, S. 236 ff.) existieren drei Generationen technologischer Innovation, die das Fernlernen bestimmen. Es handelt sich erstens um die Korrespondenz-Generation ab 1728, in der Printmedien versendet wurden, in der Regel allerdings noch unidirektional und ohne Interaktion zwischen Lehrenden und Lernenden. Die zweite Entwicklung stellt die Telekommunikations-Generation dar, in der viele Fernuniversitäten gegründet wurden, so auch die Open University (1969) in Großbritannien und die Fernuniversität Hagen (1974). In dieser Zeit implementierten die Fernstudiengänge Kommunikationsmöglichkeiten per Telefon und Fax oder auch Videokonferenz. Das Fernstudium blieb allerdings unter Lehrenden außerhalb der Fernhochschulen relativ unbekannt (Bierfelder und Müller Philipps Sohn 1974, S. 172). Die dritte Generation entstand durch die neuen Möglichkeiten von Computer und Internet, in der auch der computergestützte Unterricht eingeführt wurde. Im weiteren Verlauf entwickelten sich die heute bekannten und weit verbreiteten Lern- und Campusmanagement-Systeme, welche neben der Informations- 
bereitstellung in Form von Text, Video oder Podcasts auch stark auf Kommunikation und Kollaboration setzen. Im Rahmen des Web 2.0 und des mobilen Lernens entstanden immer mehr Instrumente zur gemeinsamen Erarbeitung von Inhalten, so zum Beispiel Wikis oder Blogs (Zawacki-Richter 2013, S. 5 f.). Der Diskurs unter den Studierenden und Lehrenden sowie die Betreuung sind besonders wichtig, da sie einen Schlüsselfaktor für den Studienerfolg im virtuellen Lernen bzw. im Fernstudium darstellen (Schulmeister 2006, S. 143 ff.), denn Fernstudiengänge weisen im Vergleich zu Präsenzstudiengängen besonders hohe Abbruchquoten auf (Brindley 2014, S. 289 ff.). Eine nähere Analyse des im Fernstudium verwendeten Blended- oder ELearning findet sich in Kapitel 4.3.

Im Wintersemester 2016/17 waren 163.653 Studierende in Fernstudiengängen eingeschrieben, davon etwa 45 Prozent in den Wirtschaftswissenschaften (Statistisches Bundesamt 2017b). Einen großen Anteil an diesen Studierenden hat die staatliche Fernuniversität Hagen, an der 65.012 Studierende in Bachelor- oder Masterstudiengängen eingeschrieben sind (Fernuniversität Hagen 2017). Wie viele Fernstudiengänge in Deutschland angeboten werden, ist nicht ganz klar; die Angaben differieren je nach Informationsquelle. Im Hochschulkompass der Hochschulrektorenkonferenz wurden im November 2017562 Fernstudiengänge gelistet, davon die Hälfte an privaten Hochschulen. Das Portal fernstudiumcheck.de hingegen bot zum gleichen Zeitpunkt 953 Studiengänge an, die zu einem Bachelor- oder Masterabschluss führen.

Fernstudiengängen ist der Lernort außerhalb der Hochschule gemein. Die Lerndauer hingegen differiert: so können Fernstudiengänge sowohl in Voll- als auch in Teilzeit angeboten werden. Häufig werden sie von Studierenden besucht, die berufsbegleitend studieren möchten. Fernstudiengänge weisen eine hohe Flexibilität auf und sind daher prinzipiell für erwerbstätige Studierende und Studierende mit Sorgeaufgaben gut geeignet. Der hohe Anteil des Selbststudiums von 75 bis 100 Prozent - im Vergleich zum Teilzeit-Präsenzstudium mit 50 bis 75 Prozent (Klumpp und Rybnikova 2010, S. 50) - stellt allerdings hohe Anforderung an die Selbstorganisation und Motivation der Studierenden.

\subsubsection{Duales Studium}

Ein duales Studium beinhaltet mindestens zwei Lernorte, in jedem Fall sind dies die Berufspraxis und die Hochschule. Dabei müssen laut der Definition des Wissenschaftsrates beide Bereiche in das Gesamtkonzept des Studiums integriert sein, was sich in Koordinierungsgremien sowie Verträgen zwischen der Hochschule, dem Ausbildungsträger bzw. dem Arbeitgeber sowie dem 
Studierenden ausdrückt (Wissenschaftsrat 2013, 22 ff.). Der Wissenschaftsrat definiert duales Studium wie folgt:

„Dualität verlangt sowohl einen angemessenen Umfang der Praxisanteile als auch eine
Verbindung und Abstimmung der Lernorte. Diese Verbindung muss strukturell mindestens
durch eine organisatorische Koordinierung der Lernorte und inhaltlich mindestens durch eine
Nähe von Studienfach und beruflicher Ausbildung/Tätigkeit gegeben sein. Eine nur zeitliche
Ermöglichung eines Studiums für Auszubildende/ Berufstätige oder ein studienbegleitendes
Praktikum bezeichnet der Wissenschaftsrat nicht als „dual“ (Wissenschaftsrat 2013, S. 22)“.

Damit sind nur integrierende Studiengänge als dual zu betrachten (Tabelle 12). Berufsbegleitende Studiengänge, die lediglich die Ausübung eines beliebigen Berufes neben dem Studium ermöglichen, sind keine dualen Studiengänge.

Tabelle 12 Typologie der dualen Studienformate laut Wissenschaftsrat (2013, S. 23). Eigene Darstellung.

\begin{tabular}{|c|c|c|}
\hline \multicolumn{2}{|c|}{ Bildungsabschnitt } & Studienformat \\
\hline \multirow{3}{*}{ 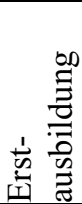 } & Mit & Ausbildungsintegrierend (Bachelor) \\
\hline & Berufsausbildung & \\
\hline & Mit Praxisanteilen & $\begin{array}{l}\text { Praxisintegrierend (Bachelor): } \\
\text { gestalteter Ausbildungsanteil beim } \\
\text { Praxispartner }\end{array}$ \\
\hline \multirow{2}{*}{ 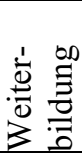 } & Mit Berufstätigkeit & $\begin{array}{l}\text { Berufsintegrierend (Bachelor/Master) } \\
\text { mit gestalteten Bezugsrahmen }\end{array}$ \\
\hline & Mit Praxisanteilen & Praxisintegrierend (Bachelor/Master) \\
\hline
\end{tabular}

Duale Studiengänge entstanden in den 1970er-Jahren, als in Baden-Württemberg die ersten Berufsakademien gegründet wurden. In den 1980er-Jahren begannen die Fachhochschulen, gemeinsam mit Unternehmen duale Studiengänge zu implementieren (Becker 2006, S. 13 f.). Seitdem ist die Anzahl der dualen Studiengänge in Deutschland immer weiter gestiegen. Wurden 2004 noch 512 duale Studiengänge angeboten, ${ }^{8}$ waren es 2014 schon 1.505 mit 94.723 eingeschriebenen Studierenden. Diese Zahlen beziehen sich auf das Erststudium, sind allerdings nicht eindeutig (BIBB 2014, 10S. ff.). Denn im November 2017 wies der Hochschulkompass nur 1.148 duale berufsintegrierende oder ausbildungsintegrierende Studiengänge im Erststudium aus. Die Zahl der dualen Studierenden scheint nahezu konstant geblieben zu sein, da im Wintersemester 2017/18 rund 92.500 Studierende (3,3 Prozent) eingeschrieben waren. Duale Studiengänge werden insbesondere in den Wirtschaftswissenschaften und in

\footnotetext{
${ }^{8} 2004$ gab es noch keine Differenzierung nach Erststudium und Weiterbildung.
} 
mathematisch- und naturwissenschaftlichen Fächern angeboten (Purz 2011, S. 89). Einen starken Anstieg gab es jedoch in den letzten Jahren auch in den Bereichen Sozialwesen, Gesundheit und Pflege (BIBB 2014, S. 9). In der Organisation von dualen Studiengängen sind besonders die abgestimmte Kompetenzentwicklung an beiden Lernorten und der hohe Betreuungsaufwand Herausforderungen für die Hochschulen (Purz 2011, S. 122 f.; Wochnik und Thiel de Gafenco o. J., S. 22 ff.).

Duale Studiengänge scheinen auf den ersten Blick eine hohe Flexibilität zu ermöglichen, da sie Zeiträume für eine berufliche Tätigkeit vorsehen und die Studienfinanzierung durch die Unternehmen in der Regel gesichert ist. Dies ist jedoch aufgrund der starken Strukturiertheit der Studienprogramme und der häufigen Nutzung der vorlesungsfreien Zeiten für die Ausbildung oder Praxisanteile nur eingeschränkt der Fall. Duale Bachelorstudiengänge (insbesondere alle Studiengänge der Dualen Hochschule Baden-Württemberg ${ }^{9}$ ) sind vergleichsweise häufig als Intensivstudiengänge mit besonders hohem Workload anerkannt, hier leisten die Studierenden 210 ECTS in sechs Semestern ab.

Zusammenfassend lässt sich konstatieren, dass viele Dualstudierende einem hohen Leistungs- und Zeitdruck unterliegen. Um diesen Druck zu bewältigen, benötigen sie ein hohes Maß an Konzentrationsfähigkeit, Zeitmanagement und Eigenverantwortlichkeit (Hesser und Langfeldt 2017, S. 38 f.). Duale Studiengänge bieten damit für Studierende, die neben dem Studium einen Beruf erlernen oder berufstätig sein möchten und gleichzeitig die finanzielle Sicherheit wertschätzen, grundsätzlich gute Bedingungen. Die notwendige Flexibilität für Studierende mit Sorgeaufgaben oder Behinderungen ist jedoch nicht garantiert und sollte im Einzelfall geprüft werden.

\subsection{Hypothesen zu Studienstrukturen}

Alle Studienstrukturen und Studienformate, welche die Bedarfe von unterschiedlichen Studierendengruppen befriedigen, sollten einen angemessenen Grad an Flexibilität beinhalten. Diese Flexibilität existiert dann, wenn die Studierenden eine möglichst freie Einteilung ihrer Zeit vornehmen können, also zwischen unterschiedlichen Lehrveranstaltungszeiten wählen und von zu Hause mit Blended-Learning studieren können. Dabei sollten ausreichend große Zeitfenster für das konzentrierte Selbststudium möglich sein. Entsprechend ist die Hypothese H1.1 zu überprüfen, die besagt, dass die in diesem Kapitel entwickelten

\footnotetext{
${ }^{9}$ Informationen zu Intensivstudiengängen an der DHBW: www.dhbw.de/informationen/studieninteressierte.html\#Vorteile.
} 
flexiblen Studienstrukturen mit geringeren Schwierigkeiten beim Besuch von Lehrveranstaltungen und Prüfungen sowie der Selbstlernzeit zusammenhängen.

\section{H1.1: Diejenigen Studienstrukturen, welche mit dem Studierverhalten positiv zusammenhängen, sind eine geringe Anzahl an SWS, hohe Wahlmöglichkeiten, eine gleichmäßige Verteilung von Prüfungen, freie Zeiträume für das Selbststudium und Blended-Learning.}

Die Hypothese H1.2 verbindet die Studienstrukturen mit Handlungstheorien und der Definition von Präferenzen (Kapitel 2.4) sowie zeitlichen Restriktionen (Kapitel 1). Dabei wird angenommen, dass Studierende mit mittleren und hohen zeitlichen Restriktionen über eine klare Präferenzordnung verfügen, die flexible Studienstrukturen unflexiblen Studienstrukturen vorzieht. Dies gilt, da Studierende mit familiären Pflichten, hoher Erwerbstätigkeit oder einer studieneinschränkenden Beeinträchtigung sich eher in einer Hochkostensituation bezüglich ihres Zeitbudgets befinden.

\section{H1.2: Studierende mit zeitlichen Restriktionen präferieren \\ Studienstrukturen und Studienformate, die ihnen zeitliche und örtliche Flexibilität ermöglichen.}

Die Ergebnisse der Hypothesenprüfung werden in Kapitel 7.4 für H1.1 und in Kapitel 8.6 für H1.2 zusammengefasst.

Open Access Dieses Kapitel wird unter der Creative Commons Namensnennung 4.0 International Lizenz (http://creativecommons.org/licenses/by/4.0/deed.de) veröffentlicht, welche die Nutzung, Vervielfältigung, Bearbeitung, Verbreitung und Wiedergabe in jeglichem Medium und Format erlaubt, sofern Sie den/die ursprünglichen Autor(en) und die Quelle ordnungsgemäß nennen, einen Link zur Creative Commons Lizenz beifügen und angeben, ob Änderungen vorgenommen wurden.

Die in diesem Kapitel enthaltenen Bilder und sonstiges Drittmaterial unterliegen ebenfalls der genannten Creative Commons Lizenz, sofern sich aus der Abbildungslegende nichts anderes ergibt. Sofern das betreffende Material nicht unter der genannten Creative Commons Lizenz steht und die betreffende Handlung nicht nach gesetzlichen Vorschriften erlaubt ist, ist für die oben aufgeführten Weiterverwendungen des Materials die Einwilligung des jeweiligen Rechteinhabers einzuholen.

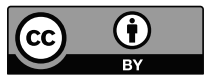

